\title{
Evaluating protocol of management of unconjugated hyperbilirubinemia in neonatal intensive care unit at Qena University Hospital
}

Eman Ahmed Abd-Elmawgood ${ }^{\mathrm{a}}$, Ahmed El-Abd Ahmed ${ }^{\mathrm{a}}$, Abdelqader Mohamed Kamel ${ }^{\mathrm{a}}$, Nagwan Ibrahim Rashwan ${ }^{\text {a }}$

${ }^{a}$ Pediatrics Department, Qena Faculty of Medicine South Vally University

Background: Neonatal hyperbilirubinemia is a common problem in neonates with an incidence of about $60 \%$ in term babies and $80 \%$ in preterm babies. It is the commonest cause of admission to the hospitals in the newborn period. We should assess all babies for jaundice at every opportunity. Methods include visual assessment, transcutaneous bilirubinometer (TcB) or total serum bilirubin (TSB).

Objective: This is a descriptive study aimed to evaluating the protocol of management of unconjugated hyperbilirubinemia in neonatal intensive care unit at Qena University Hospital.

Patients and method(s): This descriptive study included neonates admitted to neonatology unit at Qena university hospital from (April to October 2019), Total number of admitted neonates in our NICU through this period were 1274, from which 517 neonates had jaundice as a primary cause of admission or developed during the course of NICU stay.

Result(s): In this period the total number of NICU admission was 1274 cases, out of 517 jaundiced neonates, $100(19.3 \%)$ were diagnosed to have unconjugated jaundice on admission without any associated other diseases and $417(80.7 \%)$ of them were admitted due to other causes and developed jaundice during the course of the disease. The other 417 neonates were admitted to our NICU due to RD in $409(79.1 \%)$ cases, $4(0.8 \%)$ cases due to sepsis, two $(0.4 \%)$ cases due to CHD and two $(0.4 \%)$ cases due to surgical causes. The unconjugated neonatal jaundice represented (7.8\%) (100 cases out of 1274 neonates admitted during the period of the study). Physiological jaundice represented $89.4 \%$ while pathological jaundice represented $10.6 \%$ of causes of neonatal jaundice. Regarding treatment of studied neonates $12 \%$ of cases need only follow up with no need for Phototherapy, $62.8 \%$ of cases needed single or double Phototherapy, $12.4 \%$ needed extensive Phototherapy (triple phototherapy), $12.8 \%$ of cases needed capsule Phototherapy and no cases needed neither exchange transfusion nor drug therapy. Only $0.4 \%$ of jaundiced neonates developed prolonged hyperbilirubinemia, while no complications were detected in $99.6 \%$ of cases. We started treatment based on measuring bilirubin by TSB in $62.3 \%$ of jaundiced neonates and $37.7 \%$ by TCB. The mean values of TSB in jaundiced neonates (11.46 \pm $4.92)$ were significantly higher than the TCB $(10.31 \pm 4.61), \mathrm{P}=0.0005$. There was significant positive correlation between mean TSB and mean TCB $(\mathrm{R}=0.946, \mathrm{P}<0.001)$. There was no significant difference regarding mean TSB between different gestational age groups $\mathrm{p}=0.242$ ).

Conclusions: Hyperbilirubinemia is one of the most common causes of hospital admission in our nursery. Among the causes of hyperbilirubinemia, physiologic jaundice was the most common. Intensive phototherapy is effective in lowering TSB in unconjugated jaundice at / or near levels of exchange transfusion, and this may be helpful in decreasing needs for and risks of exchange transfusions. TcB measurements may underestimate the TSB values, so it should be considered only as a screen and samples should be sent to the laboratory for confirmation especially at high risk groups and high levels of TCB.

Keywords: Neonatal Jaundice, Hyperbilirubinemia, Transcutaneous bilirubin, Qena.

\section{Introduction:}

Neonatal hyperbilirubinemia is a common problem in neonates with an incidence of about $60 \%$ in term babies and $80 \%$ in preterm babies. It is the commonest cause of admission to the
Neonatal jaundice may be physiological or pathological (Martin and Cloherty, 2007). 
We should assess all babies for jaundice at every opportunity. Methods include visual assessment, transcutaneous bilirubinometer (TcB) or total serum bilirubin (TSB). TCB can be a suitable tool for predicting neonatal icterus. However, it is not a suitable substitute for TSB measurement in neonates at a high risk of jaundice (Majid Mansouri et al., 2015).

The adequacy of breastfeeding, weight $\&$ hydration status of all babies should be assessed during the first week of life. Refer babies with weight loss $\geq 7 \%$ of birth weight for further evaluation. Start phototherapy when TSB reaches the phototherapy threshold. The threshold is lower in preterm \& low birth weight babies. This should follow a standardized protocol \& be supervised by experienced personnel. Babies discharged <48 hours after birth should be seen by a healthcare provider in an ambulatory setting or at home within 24 hours of discharge. Continue breastfeeding in babies with jaundice. Provide adequate lactation support to all mothers, particularly those with preterm babies (MaHTAS. 2014).

The use of Bilisphere 360 in the treatment of indirect pathological hyperbilirubinemia is as effective as exchange transfusion in lowering Total Serum Bilirubin when its level is within 2-3 mg/dl (34-51umol/l) of the exchange level (Amira Abdel Fattah et al., 2014).

\section{Patient and Methods:}

This descriptive study included neonates admitted at neonatology unit of Qena university hospital from (April to October 2019), Total number of admitted neonates in our NICU through this period was 1274, from which 517 neonates had jaundice as a primary cause of admission or developed jaundice during the course of NICU stay.

\section{1- Inclusion criteria:-}

All neonates attended neonatal intensive care unit at Qena University Hospital who discovered to have unconjugated hyperbilirubinemia at that period.

\section{2-All patients were subjected to the following:-}

\section{1) Medical history:-}

All neonates in this study were subjected to the following data:

1. Gestational age.

2. Postnatal age.

3. Age at which jaundice firstly appear.

4. Sex.

5. Obstetric history.

6. Family history of neonatal jaundice

2) Clinical examination:-

Clinical examination (general and systematic).

\section{3) Investigations:-}

The following tests were carried out:

1- Neonatal blood group \& Rh: done by using antisera by slide and tube method.

2- Complete blood count (CBC)

3- Reticulocytic count: using methylene blue stain.

4- Transcutaneous bilirubinometer Three ml blood taken on plain vacutainer left to clot and centrifuged to separate serum which is used for liver function tests: Serum bilirubin (total and indirect) albumin and blood culture when indicated.

4) Management of NJ regarding phototherapy and exchange transfusion was according to American academy of pediatrics guidelines (American academy of pediatrics. 2004)

\section{Statistical analysis:}

Data were analyzed using Statistical Package for Social Sciences (SPSS) software program (version 20). Qualitative variables were recorded as frequencies and percentages and were compared by chi-square test. Quantitative measures were presented as means \pm standard deviation (SD).

Probability (P-value):

-P-value $<0.05$ was considered significant.

P-value $<0.001$ was considered as highly significant.-

-P-value >0.05 was considered insignificant. 


\section{Results:}

This was a descriptive study of all neonates attended neonatal intensive care unit at Qena university hospital that were diagnosed unconjugated hyperbilirubinemia between April 2019 and October 2019. In this period 517 neonates admitted to our unit. Regarding demographic data of included neonates, $(56.1 \%)$ of jaundiced neonates were males and $(43.9 \%)$ were females. Regarding type of delivery, (21.1\%) of neonates delivered by NVD and (78.9\%) by CS. Regarding duration of hospital staying, $(55.3 \%)$ of cases stayed for < 7days, $(29.6 \%)$ of cases stayed for 7-14 days, (13.9\%) of cases stayed for 14-28 days, while (1.2\%) of cases stayed for more than 28 days. As regards gestational age, $(1.2 \%)$ of neonates were $<30$ wks, (24\%) of neonates were 31-35 wks, (74.8\%) of neonates were $36-40$ wks. Regarding type of feeding, only (2.9\%) was breast fed, $(69.4 \%)$ of neonates were formula fed. Regarding body weight, (1\%) of cases were $<1$ $\mathrm{kg},(20.7 \%)$ of cases were $1-1.5 \mathrm{~kg},(49.9 \%)$ of cases were $1.5-2.5 \mathrm{~kg}$ and $(28.4 \%)$ of cases were $2.5-4 \mathrm{~kg}$. As regards type of birth, most of neonates were single $(91.5 \%)$ and only $(9.5 \%)$ of neonates were twins (table 1).

Table (1): Demographic data of the studied neonates

\begin{tabular}{|l|l|l|}
\hline & No. (517) & \% \\
\hline Sex: & & \\
\hline Male & 290 & 56.1 \\
\hline Female & 227 & 43.9 \\
\hline \hline Type of delivery: & & \\
\hline NVD & 109 & 21.1 \\
\hline CS & 408 & 78.9 \\
\hline \hline Hospital staying: & & \\
\hline < days & 286 & 55.3 \\
\hline 7 - 14 days & 153 & 29.6 \\
\hline $14-28$ days & 72 & 13.9 \\
\hline$>28$ days & 6 & 1.2 \\
\hline \hline Gestational age: & & \\
\hline < 30 weeks & 6 & 1.2 \\
\hline $30-35$ weeks & 124 & 24.0 \\
\hline $36-40$ weeks & 387 & 74.8 \\
\hline \hline Place of birth: & & \\
\hline Home & 38 & 7.4 \\
\hline Hospital & 447 & 86.4 \\
\hline Private clinic & 32 & 6.2 \\
\hline \hline Type of feeding: & & \\
\hline Breast feeding & 15 & 2.9 \\
\hline Formula & 359 & 69.4 \\
\hline Mixed & 6 & 1.2 \\
\hline
\end{tabular}

\begin{tabular}{|l|l|l|}
\hline TPN & 137 & 26.5 \\
\hline \hline Body weight: & & \\
\hline$<1 \mathrm{~kg}$ & 5 & 1.0 \\
\hline $1.0-1.5 \mathrm{~kg}$ & 107 & 20.7 \\
\hline $1.5-2.5 \mathrm{~kg}$ & 258 & 49.9 \\
\hline $2.5-4.5 \mathrm{~kg}$ & 147 & 28.4 \\
\hline \hline Type of birth: & & \\
\hline Single & 473 & 91.5 \\
\hline Twins & 44 & 8.5 \\
\hline
\end{tabular}

Through the period from April 2019 to October 2019, the total number of admission in NICU was 1274 cases, from which, 517 neonates had neonatal jaundice with indirect hyperbilirubinemia. Out of 517 Jaundiced neonates, $100 \quad(19.3 \%)$ neonates had unconjugated neonatal jaundice as a primary cause of admission and 417 (80.7\%) of cases were admitted due to other causes and developed jaundice during the course of the disease. The causes of admission in the other 417 cases were $\mathrm{RD}$ in $409(79.1 \%)$ cases, sepsis in $4(0.8 \%)$, CHD in two $(0.4 \%)$ cases and surgical causes in two $(0.4 \%)$ cases. The unconjugated neonatal jaundice on admission without any associated diseases represented (7.8\%) (100 cases out of 1274 neonates admitted during the period of the study) (Table 2).

Table (2): Type of jaundice and associated diseases

\begin{tabular}{|l|l|l|}
\hline & No. (517) & \% \\
\hline Diagnosis on admission: & & \\
\hline Isolated NJ & 100 & 19.3 \\
\hline RD & 409 & 79.1 \\
\hline CHDs & 2 & 0.4 \\
\hline Surgical & 2 & 0.4 \\
\hline Sepsis & 4 & 0.8 \\
\hline Type of jaundice: & & \\
\hline Physiological & 462 & 89.4 \\
\hline Pathological & 55 & 10.6 \\
\hline \hline $\begin{array}{l}\text { Causes of pathological } \\
\text { jaundice: }\end{array}$ & & \\
\hline ABO incompatibility & 33 & 60.0 \\
\hline Rh incompatibility & 18 & 32.7 \\
\hline Sepsis & 4 & 7.3 \\
\hline
\end{tabular}

RD (respiratory distress), CHD (congenital heart diseases).

Regarding the age of onset of jaundice most of neonates develop $\mathrm{NJ}$ after 3 days postnatal (88\%). Regarding method of detection of NJ, $(72.5 \%)$ of cases were detected in NICU, (14.5 $\%$ ) detected by the family and only $(5.1 \%)$ of neonates detected by outpatient clinic. Only $0.4 \%$ of jaundiced neonates developed prolonged 
hyperbilirubinemia, while no complications were detected in $99.6 \%$ of cases (table 3).

Table (3): Clinical data of jaundiced neonates

\begin{tabular}{|c|l|l|}
\hline & No. (517) & \% \\
\hline Age of onset: & & \\
\hline Day 1 & 6 & 1.2 \\
\hline Day 2 & 20 & 3.8 \\
\hline Day 3-7 & 455 & 88.0 \\
\hline Day > 7 & 36 & 7.0 \\
\hline \hline $\begin{array}{l}\text { Method of Neonatal } \\
\text { jaundice detection: }\end{array}$ & & \\
\hline \hline Hospital & 41 & 7.9 \\
\hline Family & 75 & 14.5 \\
\hline Outpatient Clinic & 26 & 5.1 \\
\hline NICU & 375 & 72.5 \\
\hline \hline Complications: & & \\
\hline No & 515 & 99.6 \\
\hline Kernicterus & 0 & 0.0 \\
\hline $\begin{array}{l}\text { Prolonged } \\
\text { hyperbilirubinemia }\end{array}$ & 2 & 0.4 \\
\hline
\end{tabular}

Regarding treatment used in the jaundiced neonates, $12 \%$ of cases need only follow up with no need for Phototherapy, $62.8 \%$ of cases need single or double Phototherapy, $12.4 \%$ need extensive Phototherapy (triple phototherapy), $12.8 \%$ of cases need capsule Phototherapy and no cases need neither exchange transfusion nor drug therapy, also we started treatment based on measuring bilirubin by TSB in $62.3 \%$ of jaundiced neonates and $37.7 \%$ by TCB (Table 4).

Table (4): Treatment used in the jaundiced neonates

\begin{tabular}{|l|l|l|}
\hline & No. (517) & \% \\
\hline Treatment given: & & \\
\hline No (Follow-up) & 62 & 12.0 \\
\hline Phototherapy & 325 & 62.8 \\
\hline Capsule & 66 & 12.8 \\
\hline Extensive phototherapy & 64 & 12.4 \\
\hline Exchange transfusion & 0 & 0.0 \\
\hline Phenobarbital & 0 & 0.0 \\
\hline $\begin{array}{l}\text { Treatment start on / } \\
\text { Bilirubin: }\end{array}$ & 195 & \\
\hline TCB & 322 & 37.7 \\
\hline TSB & 62.3 \\
\hline
\end{tabular}

Regarding the risk factors for developing NJ we found that prematurity <38 wks represented $(25.1 \%)$ of the risk factors and $(61.9 \%)$ of neonates developed $\mathrm{NJ}$ were without any detected risk factors. we also reported that $(97.9 \%)$ of neonates receiving treatment were discharged and only (2.9\%) of neonates died from other causes than unconjugated jaundice, from which, $66.7 \%$ died from RD with its different causes and complications (as Pneumonia, RDS), $26.7 \%$ from sepsis and $6.7 \%$ from CHD. In a follow -up visit after 3 days of discharge, there was complete recovery for all discharged neonates, with no reported readmission of cases (table 5).

Table (5): Risk factors and outcome of studied neonates

\begin{tabular}{|c|l|l|}
\hline & No.(517) & \% \\
\hline Risk factors: & & \\
\hline No & 320 & 61.9 \\
\hline $\begin{array}{l}\text { Jaundice observed in the } \\
\text { first 24 hours }\end{array}$ & 6 & 1.2 \\
\hline Cephalohematoma & 10 & 1.9 \\
\hline ABO, Rh incompatibility & 51 & 9.9 \\
\hline <38 weeks & 130 & 25.1 \\
\hline \hline Outcome: & & \\
\hline Death & 15 & 2.9 \\
\hline Discharge & 502 & 97.1 \\
\hline Cause of death: & & \\
\hline RD & 10 & 66.6 \\
\hline Sepsis & 4 & 26.7 \\
\hline CHD & 1 & 6.7 \\
\hline \hline Readmission after 3 days: & & \\
\hline 1. Complete recovery & 517 & 100.0 \\
\hline 2. Readmission & 0 & 0.0 \\
\hline
\end{tabular}

The mean values of TSB in jaundiced neonates $(11.46 \pm 4.92)$ was significantly higher than the TCB $(10.31 \pm 4.61), \mathrm{P}=0.0003$ (table 6).

There was significant positive correlation between mean TSB and mean TCB $(\mathrm{R}=0.946, \mathrm{P}$ $<0.001$ ) (figure 1).

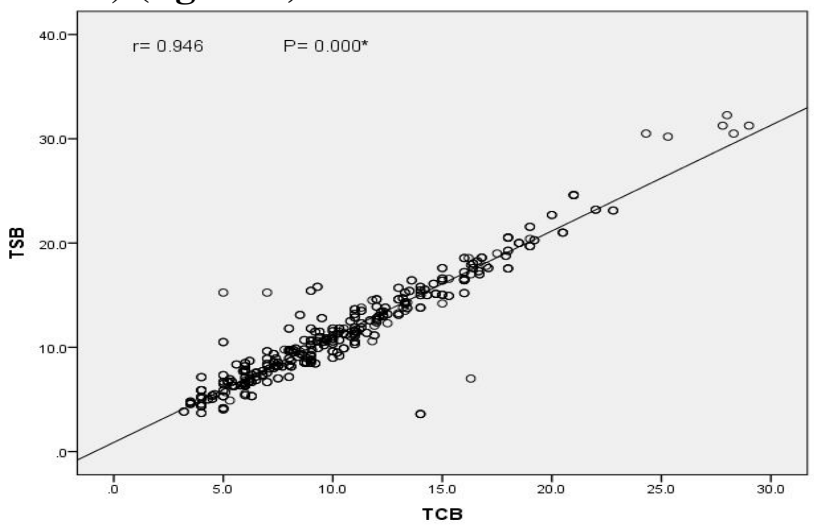

Figure (1): Correlation between TSB and TCB and mean TCB $(\mathrm{R}=0.946, \mathrm{P}<0.001)$. 
Table (6): T-test between TSB and TCB.

\begin{tabular}{|l|l|l|l|}
\hline & TSB & TCB & P-value \\
\hline Mean \pm SD & $11.46 \pm 4.92$ & $10.31 \pm 4.61$ & \multirow{2}{*}{$0.0003^{* *}$} \\
\hline Range & $3.6-32$ & $3-29$ & \\
\hline
\end{tabular}

The mean TSB levels were significantly higher among those babies who were discharged in comparison to those who died $[11.44 \pm 4.95$ vs $7.79 \pm 2.86$ respectively, with $\mathrm{p}=0.005$ ] (table 7).

Table (7): Relation between TSB and outcome

\begin{tabular}{|l|l|l|l|}
\hline \multirow{2}{*}{ TSB } & \multicolumn{2}{|c|}{ Outcome } & \multirow{2}{*}{$\begin{array}{l}\text { P- } \\
\text { value }\end{array}$} \\
\cline { 2 - 3 } & Death & Discharge & \multirow{2}{*}{$0.005^{*}$} \\
\hline Mean \pm SD & $7.79 \pm 2.86$ & $11.44 \pm 4.95$ & \\
\hline Range & $3.8-13.4$ & $3.6-32.3$ & \\
\hline
\end{tabular}

\section{Discussion:}

Neonatal jaundice refers to the yellowish discoloration of the skin and sclera of newborn babies that result from accumulation of bilirubin in the skin and mucus membranes. Clinically it becomes apparent when the serum bilirubin exceeds $7 \mathrm{mg} / \mathrm{dl}$ (Ali and Tomar, 2015). Neonatal jaundice may be physiological or pathological (Martin and Cloherty, 2007).

Neonatal jaundice is still a problem responsible for significant neonatal morbidity despite great advances in neonatal medicine. In our study the total number of admission in NICU was 1274 cases from which, 517 neonates had neonatal jaundice, out of them 100 (19.3\%) neonates had unconjugated neonatal jaundice on admission without any association of other diseases and 417 (80.7\%) of cases were admitted due to other causes and developed jaundice during the course of the disease. The unconjugated neonatal jaundice represented (7.8\%) (100 cases out of 1274 neonates admitted during the period of the study). The other 417 neonates were admitted to our NICU due to RD in $409(79.1 \%)$ cases, $4(0.8 \%)$ cases due to sepsis, two $(0.4 \%)$ cases due to CHD and two $(0.4 \%)$ cases due to surgical causes.

Similar to our findings in a recent study by (Siyal et al., 2019) on the neonatal admissions in the Neonatal Unit of Pediatric Ward of PMC Hospital, Nawabshah, there was a total of 194 neonates were found to have hyperbilirubinemia, out of 2863 babies admitted during this period, representing $6.8 \%$ of the total admissions. Nepal et al., 2010 performed a retrospective observational study on the neonates admitted in a tertiary care paediatric hospital (Kanti Children Hospital) and they reported that out of 361 neonatal admissions, 73 babies (20\%) were admitted with the diagnosis of neonatal jaundice (NJ) and total serum bilirubin level $>15$ $\mathrm{mg} / \mathrm{dl}$ which constituted $20 \%$ of neonatal morbidity.

In the present study, we found that males represented $(56.1 \%)$ of total cases. This was in agreement with the study done by (Mantani et al., 2007) in which male represented $62 \%$. Also it was in agreement with (Ali and Tomar, 2015) who reported that the incidence of neonatal jaundice was higher in males (52\%) than in females $(48 \%)$ and probable explanation for this result may be due to social bias, males being more cared for, and promptly brought to medical attention.

In our study, there were $25.2 \%$ preterm babies and $74.8 \%$ full term babies. This was in agreement with (Ali Akbar et al., 2018) who reported that $28.8 \%$ of jaundiced neonates were preterms and $71.2 \%$ were fullterms This also was in agreement with (Shah et al., 2012) who reported that percentage of Pre-term babies was $30.1 \%$. In a study done by (Rasul et al., 2010) percentage of preterm babies was slightly higher than that in our study (37\%) but still less than full-term babies $(63 \%)$. But our study was in disagreement with (Ali and Tomar; 2015) who reported that preterm babies represented $51 \%$ and full-term represented $49 \%$.

Regarding the age at onset of $\mathrm{NJ}$, we found that first day neonatal jaundice developed in 6 (1.2) \% neonates, (Najib et al., 2013) reported that out of 170 neonatal admissions, 19 neonate (11.4) developed jaundice in first 24 hours after birth. 137 neonate $(73.5 \%)$ developed jaundice after discharging from hospital and that was in disagreement with our study. This may be attributed to different sample size, higher percent of physiological jaundice in our study than pathological jaundice, different environmental factors and higher percent of fullterms than preterms in our study.

In our study, the most common cause of neonatal jaundice was physiological jaundice in $89.4 \%$ of cases. Similar results were found by 
(Siyal et al., 2019) who reported that among the causes of hyperbilirubinemia physiologic jaundice was most common.

ABO incompatibility represented $60 \%$ of causes of pathological jaundice, $\mathrm{Rh}$ incompatibility represented $32.7 \%$ and sepsis represented $7.3 \%$ of causes of pathological jaundice. This was in agreement with (Faris B. AL-Swaf et al., 2009) who reported that ABOincompatibility is the most common cause of hemolytic diseases of newborns. (Najib et al., 2013) found in their study, that the cause of severe hyperbilirubinemia was $\mathrm{ABO}$ and $\mathrm{Rh}$ incompatibility (5.9\%), G6PD deficiency $(25.5 \%)$, sepsis $(12 \%)$, cephalohematoma (4.7 $\%)$ and unknown (50\%).

In our study according to guidelines which used for management of neonatal hyperbilirubinemia in our unit (American academy of pediatrics. 2004), we reported $12 \%$ of cases with no need for phototherapy, (62.8\%) of cases needed phototherapy, (12.4\%) of cases need extensive phototherapy (triple phototherapy) and (12.8\%) of cases need capsule phototherapy with no need for exchange transfusion, this was due to the early detection of hyperbilirubinemia and the early use of capsule phototherapy. This was in agreement with (Amira Abdel Fattah et al., 2014) who reported that the use of Bilisphere 360 in the treatment of indirect pathological hyperbilirubinemia is as effective as exchange transfusion in lowering total Serum Bilirubin when its level is within 2-3 $\mathrm{mg} / \mathrm{dl}$ (34-51umol/l) of the exchange level. Bilisphere 360 is effective in reducing needs for exchange transfusion and duration of phototherapy.

Risk factors of neonatal jaundice in our study included prematurity in 130 (25.1\%) neonates, 51(9.9\%) neonates suffering from $\mathrm{ABO}, \mathrm{Rh}$ incompatibility, (1.9\%) suffering from cephalohematoma and only $(1.2 \%)$ of neonates developed jaundice in first 24 hours after birth. Najib KS et al., 2013 reported that (4.7\%) of neonates suffering from cephalohematoma and (11.4\%) neonates developed jaundice in first 24 hours after birth.

Our study showed that most of the cases (97.1\%) discharged with another visit after 3 days with complete recovery and only $2.9 \%$ died from another cause like RD (66.6\%), sepsis (26.7\%) and CHD (6.7\%), (Rasul CHet al., 2010) reported a higher percentage of those who died from another conditions $(18.2 \%)$ than our study and this may be attributed to different environmental factors.

In our study, the TSB was significantly higher in the survivors than in non survivors who indicate that the mortality in our study was related to other causes other than $\mathrm{NJ}$, And that higher TSB has negative effect on the mortality outcome of NICU patients in our patients. Similar to our results, (Mantani et al., 2007) found that peak serum bilirubin or total serum bilirubin did not have any correlation with the final outcome.

Also similar to our study (Siyal et al., 2019) in Bangaladesh reported that twelve (2.8\%) patients in his study died in the hospital and four $(0.9 \%)$ neonates were discharged with neurological sequels.

Transcutaneous bilirubinometer (TCB) testing has become more popular than visual assessment because of the known limitations of visual identification of hyperbilirubinemia, especially in non-white babies. When used properly, however, TCB measurement appears to be reliable in identification of hyperbilirubinemia in neonates from variety of ethnic backgrounds.

In our study we reported a significant positive correlation between TSB and TCB levels. However, the mean TCB levels were significantly lower than the actual TSB values, which indicate that TCB measurements may underestimate the TSB values. Similar to our findings, (Maisels et al., 2004) stated that although a linear relationship exists between $\mathrm{TcB}$ and TSB $(r=0.87-0.96)$, at TSB concentrations > $257 \mathrm{mmol} / \mathrm{L}(15 \mathrm{mg} / \mathrm{dL})$ the accuracy of TcB has been questioned.

\section{Conclusions:}

Early detection with proper management of neonatal hyper-bilirubinemia following the guidelines with proper use of phototherapy techniques is mandatory to avoid serious complications of neonatal hyperbilirubinemia and improve outcome. 
Phototherapy is effective in most of the time but, intensive phototherapy is effective in lowering TSB in unconjugated jaundice at / or near levels of exchange transfusion, and this may be helpful in decreasing needs for and risks of exchange transfusions especially when there are no facilities to perform exchange transfusion.

TCB measurements may underestimate the TSB values, so it should be considered only as a screen and samples should be sent to the laboratory for confirmation especially at high risk groups and high levels of TCB.

\section{Recommendations}

Screening all newborns before discharge from maternity hospital and check level on hour specific bilirubin normogram is required for proper follow up and to detect high risk groups.

Although measurement of TSB remains the gold standard for assessment of neonatal jaundice, TcB is a viable option for universal screening.

Further longitudinal comparative studies between use of TSB and TCB are required to clarify the benefits and risks of use of TCB alone and to determine a cutoff point at which we should do laboratory sample for TSB.

Further longitudinal comparative studies between the use of exchange transfusion and capsule phototherapy to clarify the benefits and risks of using capsule phototherapy alone at high levels of TSB especially when the facilities of exchange transfusion are not present.

\section{References:}

1. Ali A. and TomarA, (2015). Etiological profile of neonatal hyperbilirubinemia in the rural area of Rajasthan. Indian Journal of Basic and Applied Medical Research Vol.4, Issue- 2, P. 223-32.

2. American academy of pediatrics, (2004). Management of hyperbilirubinemia in the newborn infants 35 or more weeks of gestation. Pediatrics Vol .114 No 1.

3. Amira Abdel Fattah Edris, Eman Abdel Ghany Abdel Ghany, Abdel Rahman Ahmed Abdel Razek, Amany Mosad Zahran, (2014). The role of intensive phototherapy in decreasing the need for exchange transfusion in neonatal jaundice Vol. 64, No. 1.

4. Bhutani VK, Johnson LH, (2004). Urgent clinical need for accurate and precise bilirubin measurements in the United States to prevent kernicterus. Clin Chem 50(3):477-80.

5. Boo N, Ishak S, (2007). Prediction of severe hyperbilirubinemia using the BiliCheck transcutaneous bilirubinometer. J Paediatr Child Health 43: 297-302.

6. Bryon JL. And Nancy DS, (2011). Hyperbilirubinemia in the Newborn. Pediatrics in Review 32-341.

7. Faris B. AL-Swaf, Rekan S. Jumaa, and Saeed IS, (2009). Hemolytic disease of newborn due to $\mathrm{ABO}$ incompatability. Tikrit Medical Journal 15 (2):70-78.

8. Leite M, Granto V, Facchini F, Marba S, (2007). Comparison of transcutaneous and plasma bilirubin measurements. J Pediatr (Rio J) 83:283- 6 .

9. Maisels MJ, McDonagh AF, (2008). Phototherapy for Neonatal Jaundice. N Engl J Med 358(9):920-28.

10. Malaysia Health Technology Assessment Section, (2014). Management of Neonatal Jaundice (Second Edition) 278-14.

11. Mantani M, Patel A, Renge R and Kulkarni H, (2007). Prognostic value of direct bilirubin in Neonatal Hyperbilirubinemia. Indian J Pediatr 79: 819-22.

12. Martin CR and Cloherty JP, (2007). Neonatal hyperbilirubinemia. In Cloherty JP, Eichenwaldec, Stark AR (Eds) Manual of neonatal care (Lippincott Wiliams and Wilkins, Philadelphia.

13. Najib KS, Saki F, Hemmati F and Inaloo S, (2013). Incidence, Risk Factors and Causes of Severe Neonatal Hyperbilirubinemia in the South of Iran (Fars Province). Iran Red Crescent Med J. Vol. 15(3): 
260-263.

14. Nepal D, Banstola D, Dhakal AK, Mishra U and Mahaseth C, (2010). Clinico-Laboratory Profile and Immediate Outcomes of Hyperbilirubinemic babies admitted in Kanti Children Hospital. Journal of Nepal Paediatric Society Vol. 30 (1).

15. Rasul CH, AbulHasan, Yasmin F, (2010). Outcome of Neonatal Hyperbilirubinemia in a Tertiary Care Hospital in Bangladesh. Malaysian J Med Sci. Apr-Jun 17(2): 40-44.

16. Reyes CA, Stednitz DR, Hahn C, Mutchie KD, McCullough SR, Kronberg K, (2008). Evaluation of the Bilichek being used on hyperbilirubinemic newborns undergoing home phototherapy. Arch Pathol Lab Med 132:684 -9.

17. Shah A, Shah CK and Shah V, (2012). Study of hematological parameters among neonates admitted with neonatal jaundice. Journal of Evolution of Medical and Dental Sciences Vol.01, Issue3, P. 205.

18. Siyal AA, Naqvi J, Taqi T, Haider S, (2019). Hyperbilirubinemia; prevalence of hyperbilirubinemia and causative factors in neonates admitted in neonatal unit pediatric ward Nawabshah. Professional Med J 26(1):128-131 
